\title{
The Cosmological Constant in Distorted Quantum Cosmology
}

\author{
Remo Garattini* \\ University of Bergamo, \\ Department of Engineering and Applied Sciences \\ Viale Marconi 5, 24044 Dalmine (Bergamo) Italy \\ I.N.F.N. - sezione di Milano, Milan, Italy \\ E-mail: remo.garattini@unibg.it
}

\begin{abstract}
We give a calculation scheme for the cosmological constant computation with the help of the Wheeler-DeWitt equation. This last one is regarded as a Sturm-Liouville problem with the cosmological constant considered as the associated eigenvalue. By fixing the ideas on a FriedmannLemaitre-Robertson-Walker line element in ordinary gravity, we apply this calculation scheme on distorted gravity. By distorted gravity, we mean all the deviations from General Relativity. We restrict our proposal on Gravity's Rainbow and Noncommutative geometry. A brief comment on Hořava-Lifshitz (HL) theory is discussed.
\end{abstract}

Frontiers of Fundamental Physics 14

15-18 July 2014

Aix Marseille University (AMU) Saint-Charles Campus, Marseille, France

\footnotetext{
* Speaker.
} 


\section{Introduction}

One of the cornerstone of Quantum Cosmology is the Wheeler-DeWitt (WDW) equation[1]. It represents the quantum version of the invariance with respect to time reparametrization. If we denote with $d \Omega_{3}^{2}=\gamma_{i j} d x^{i} d x^{j}$ the line element on the three-sphere, with $N$ the lapse function and with $a(t)$ the scale factor, the Friedmann-Lemaitre-Robertson-Walker line element assumes the form

$$
d s^{2}=-N^{2} d t^{2}+a^{2}(t) d \Omega_{3}^{2} .
$$

Thus the WDW in absence of matter fields is

$$
\begin{aligned}
H \Psi(a) & =\left[-a^{-q}\left(\frac{\partial}{\partial a} a^{q} \frac{\partial}{\partial a}\right)+\frac{9 \pi^{2}}{4 G^{2}}\left(a^{2}-\frac{\Lambda}{3} a^{4}\right)\right] \Psi(a) \\
& =\left[-\frac{\partial^{2}}{\partial a^{2}}-\frac{q}{a} \frac{\partial}{\partial a}+\frac{9 \pi^{2}}{4 G^{2}}\left(a^{2}-\frac{\Lambda}{3} a^{4}\right)\right] \Psi(a)=0,
\end{aligned}
$$

where we have also introduced a factor order ambiguity $q$, the Newton's constant $G$ and a cosmological constant $\Lambda$. In this formulation all the degrees of freedom except the scale factor $a(t)$ have been integrated. If the WDW equation is interpreted as an eigenvalue equation, one simply finds

$$
H \Psi(a)=E \Psi(a)=0
$$

namely a zero energy eigenvalue. However, it appears that the WDW equation has also a hidden structure. Indeed Eq.(1.2) has the structure of a Sturm-Liouville eigenvalue problem with the cosmological constant as eigenvalue. We recall to the reader that a Sturm-Liouville differential equation is defined by

$$
\frac{d}{d x}\left(p(x) \frac{d y(x)}{d x}\right)+q(x) y(x)+\lambda w(x) y(x)=0
$$

and the normalization is defined by

$$
\int_{a}^{b} d x w(x) y^{*}(x) y(x),
$$

where the boundary conditions are momentarily suspended. It is a standard procedure, to convert the Sturm-Liouville problem (1.4) into a variational problem of the form

$$
F[y(x)]=\frac{-\int_{a}^{b} d x y^{*}(x)\left[\frac{d}{d x}\left(p(x) \frac{d}{d x}\right)+q(x)\right] y(x)}{\int_{a}^{b} d x w(x) y^{*}(x) y(x)},
$$

with unspecified boundary condition. If $y(x)$ is an eigenfunction of (1.4), then

$$
\lambda=\frac{-\int_{a}^{b} d x y^{*}(x)\left[\frac{d}{d x}\left(p(x) \frac{d}{d x}\right)+q(x)\right] y(x)}{\int_{a}^{b} d x w(x) y^{*}(x) y(x)},
$$

is the eigenvalue, otherwise

$$
\lambda_{1}=\min _{y(x)} \frac{-\int_{a}^{b} d x y^{*}(x)\left[\frac{d}{d x}\left(p(x) \frac{d}{d x}\right)+q(x)\right] y(x)}{\int_{a}^{b} d x w(x) y^{*}(x) y(x)} .
$$


The minimum of the functional $F[y(x)]$ corresponds to a solution of the Sturm-Liouville problem (1.4) with the eigenvalue $\lambda$. In the case of the FLRW model we have the following correspondence

$$
\begin{aligned}
p(x) & \rightarrow a^{q}(t), \\
q(x) & \rightarrow\left(\frac{3 \pi}{2 G}\right)^{2} a^{q+2}(t), \\
w(x) & \rightarrow a^{q+4}(t), \\
y(x) & \rightarrow \Psi(a), \\
\lambda & \rightarrow \frac{\Lambda}{3}\left(\frac{3 \pi}{2 G}\right)^{2} .
\end{aligned}
$$

Since $a(t) \in[0, \infty)$, the normalization becomes

$$
\int_{0}^{\infty} d a a^{q+4} \Psi^{*}(a) \Psi(a),
$$

where it is understood that $\Psi(\infty)=0$. In the minisuperspace approach with a FLRW background, one finds

$$
\frac{\int \mathscr{D} a a^{q} \Psi^{*}(a)\left[-\frac{\partial^{2}}{\partial a^{2}}-\frac{q}{a} \frac{\partial}{\partial a}+\frac{9 \pi^{2}}{4 G^{2}} a^{2}\right] \Psi(a)}{\int \mathscr{D} a a^{q+4} \Psi^{*}(a) \Psi(a)}=\frac{3 \Lambda \pi^{2}}{4 G^{2}} .
$$

As a concrete case, fixing $q=0$ and taking as a trial wave function $\Psi(a)=\exp \left(-\beta a^{2}\right)$, one finds $\Psi(a) \rightarrow 0$ when $a \rightarrow \infty$. Then the only solution allowed is complex and therefore it must be discarded[2]. Of course, the general $q$ case is much more complicated[3]. Note that the global energy eigenvalue is still vanishing. What we can compute in the Sturm-Liouville formulation is the degree of degeneracy which is represented by the cosmological constant and the value of the cosmological constant itself. In the next section we give the general guidelines in ordinary gravity and in presence of Modified Dispersion Relations and the Non Commutative approach to Quantum Field Theory. Units in which $\hbar=c=k=1$ are used throughout the paper.

\section{The Cosmological Constant in Distorted Quantum Cosmology}

When we generalize the minisuperspace approach of Eq.(1.11), the formal structure persists. Indeed the WDW equation can be transformed into $(\kappa=8 \pi G)$

$$
\frac{1}{V} \frac{\int \mathscr{D}\left[g_{i j}\right] \Psi^{*}\left[g_{i j}\right] \int_{\Sigma} d^{3} x \hat{\Lambda}_{\Sigma} \Psi\left[g_{i j}\right]}{\int \mathscr{D}\left[g_{i j}\right] \Psi^{*}\left[g_{i j}\right] \Psi\left[g_{i j}\right]}=\frac{1}{V} \frac{\left\langle\Psi\left|\int_{\Sigma} d^{3} x \hat{\Lambda}_{\Sigma}\right| \Psi\right\rangle}{\langle\Psi \mid \Psi\rangle}=-\frac{\Lambda}{\kappa},
$$

where we have integrated over the hypersurface $\Sigma$ and we have defined

$$
V=\int_{\Sigma} d^{3} x \sqrt{g}
$$

as the volume of the hypersurface $\Sigma$ with

$$
\hat{\Lambda}_{\Sigma}=(2 \kappa) G_{i j k l} \pi^{i j} \pi^{k l}-\sqrt{g} R /(2 \kappa) .
$$

$G_{i j k l}$ is the supermetric, while $\pi^{i j}$ is termed the supermomentum, $R$ is the three scalar curvature and $\sqrt{g}$ is the determinant of the three metric $g_{i j}$. In this form, Eq.(2.1) can be used to compute Zero 
Point Energy (ZPE) provided that $\Lambda / \kappa$ be considered as an eigenvalue of $\hat{\Lambda}_{\Sigma}$, namely the WDW equation is transformed into an expectation value computation. Nevertheless, solving Eq.(2.1) is a quite impossible task, therefore we are oriented to use a variational approach with trial wave functionals. The related boundary conditions are dictated by the choice of the trial wave functionals which, in our case, are of the Gaussian type: this choice is justified by the fact that ZPE should be described by a good candidate of the "vacuum state". However if we change the form of the wave functionals we change also the corresponding boundary conditions and therefore the description of the vacuum state. It is better to observe that the obtained eigenvalue $\Lambda / \kappa$, it is far to be a constant, rather it will be dependent on some parameters and therefore it will be considered more like a "dynamical cosmological constant". Usually, when we compute Eq.(2.1) to one loop or higher loops, UltraViolet divergences appear. In ordinary gravity, to take under control such divergencies we need a regularization/renormalization scheme[4]. However, as shown by Hořava, a modification of Einstein gravity motivated by the Lifshitz theory in solid state physics[5], allows the theory to be power-counting ultraviolet (UV) renormalizable with the prescription to recover general relativity in the infrared (IR) limit. Nevertheless, Hořava-Lifshitz (HL) theory is noncovariant. Another proposal comes from Gravity's Rainbow which distorts the metric tensor around and beyond the Planck scale[6]. The basic ingredient is the definition of two arbitrary functions: $g_{1}\left(E / E_{\mathrm{Pl}}\right)$ and $g_{2}\left(E / E_{\mathrm{Pl}}\right)$, which have the following property

$$
\lim _{E / E_{\mathrm{Pl}} \rightarrow 0} g_{1}\left(E / E_{\mathrm{Pl}}\right)=1 \quad \text { and } \quad \lim _{E / E_{\mathrm{Pl}} \rightarrow 0} g_{2}\left(E / E_{\mathrm{Pl}}\right)=1 .
$$

$g_{1}\left(E / E_{\mathrm{Pl}}\right)$ and $g_{2}\left(E / E_{\mathrm{Pl}}\right)$ distort the metric in the following way. For a spherically symmetric metric, we can define

$$
d s^{2}=-\frac{N^{2}(r)}{g_{1}^{2}\left(E / E_{\mathrm{Pl}}\right)} d t^{2}+\frac{d r^{2}}{\left(1-\frac{b(r)}{r}\right) g_{2}^{2}\left(E / E_{\mathrm{Pl}}\right)}+\frac{r^{2}}{g_{2}^{2}\left(E / E_{\mathrm{Pl}}\right)}\left(d \theta^{2}+\sin ^{2} \theta d \phi^{2}\right),
$$

where $N(r)$ is known as the lapse function and $b(r)$ is the shape function. For black holes and wormholes, one has the further condition $b\left(r_{t}\right)=r_{t}$ and therefore $r \in\left[r_{t},+\infty\right)$. This is not trivial, because for instance for Dark Energy Stars, the radius $r \in[0,+\infty)$ for $b(r)$ [7]. On the other hand for a FLRW metric, we can write

$$
d s^{2}=-\frac{N^{2}(t)}{g_{1}^{2}\left(E / E_{\mathrm{Pl}}\right)} d t^{2}+\frac{a^{2}(t)}{g_{2}^{2}\left(E / E_{\mathrm{Pl}}\right)} d \Omega_{3}^{2} .
$$

Of course, ordinary gravity is recovered when $E / E_{\mathrm{Pl}} \rightarrow 0$. In a series of papers[8, 9, 10], it has been shown that Gravity's Rainbow can keep under control UV divergences, at least to one loop. This procedure has been widely tested on a spherically symmetric background. If one considers perturbations of the metric (2.5), one finds that Eq.(2.1) becomes

$$
\frac{g_{2}^{3}\left(E / E_{\mathrm{Pl}}\right)}{\tilde{V}} \frac{\left\langle\Psi\left|\int_{\Sigma} d^{3} x \tilde{\Lambda}_{\Sigma}\right| \Psi\right\rangle}{\langle\Psi \mid \Psi\rangle}=-\frac{\Lambda}{\kappa}
$$

with

$$
\tilde{\Lambda}_{\Sigma}=(2 \kappa) \frac{g_{1}^{2}\left(E / E_{\mathrm{Pl}}\right)}{g_{2}^{3}\left(E / E_{\mathrm{Pl}}\right)} \tilde{G}_{i j k l} \tilde{\pi}^{i j} \tilde{\pi}^{k l}-\frac{\sqrt{\tilde{g}} \tilde{R}}{(2 \kappa) g_{2}\left(E / E_{\mathrm{Pl}}\right)} .
$$


The symbol " $\sim$ " indicates the quantity computed in absence of rainbow's functions $g_{1}\left(E / E_{\mathrm{Pl}}\right)$ and $g_{2}\left(E / E_{\mathrm{Pl}}\right)$. Extracting the TT tensor contribution from Eq.(2.7), we find that the total one loop energy density for the graviton, or ZPE, induces a dynamical cosmological constant whose form is

$$
\frac{\Lambda}{8 \pi G}=-\frac{1}{3 \pi^{2}} \sum_{i=1}^{2} \int_{E^{*}}^{+\infty} E_{i} g_{1}\left(E / E_{\mathrm{Pl}}\right) g_{2}\left(E / E_{\mathrm{Pl}}\right) \frac{d}{d E_{i}} \sqrt{\left(\frac{E_{i}^{2}}{g_{2}^{2}\left(E / E_{\mathrm{Pl}}\right)}-m_{i}^{2}(r)\right)^{3}} d E_{i},
$$

where we have defined two r-dependent effective masses $m_{1}^{2}(r)$ and $m_{2}^{2}(r)$, corresponding to the two degrees of freedom of the graviton,

$$
\left\{\begin{array}{l}
m_{1}^{2}(r)=\frac{6}{r^{2}}\left(1-\frac{b(r)}{r}\right)+\frac{3}{2 r^{2}} b^{\prime}(r)-\frac{3}{2 r^{3}} b(r) \\
m_{2}^{2}(r)=\frac{6}{r^{2}}\left(1-\frac{b(r)}{r}\right)+\frac{1}{2 r^{2}} b^{\prime}(r)+\frac{3}{2 r^{3}} b(r)
\end{array}(r \equiv r(x)) .\right.
$$

We have to observe that only appropriate choices of $g_{1}\left(E / E_{\mathrm{Pl}}\right)$ and $g_{2}\left(E / E_{\mathrm{Pl}}\right)$ lead to a finite induced cosmological constant. For instance, if we choose

$$
g_{1}\left(E / E_{\mathrm{Pl}}\right)=1-\eta\left(E / E_{\mathrm{Pl}}\right)^{n} \quad \text { and } \quad g_{2}\left(E / E_{\mathrm{Pl}}\right)=1,
$$

where $\eta$ is a dimensionless parameter and $n$ is an integer[11], the ZPE of Eq.(2.9) diverges and therefore will be discarded. On the other hand, if we choose

$$
g_{1}\left(E / E_{\mathrm{Pl}}\right)=\left(1+\beta \frac{E}{E_{\mathrm{Pl}}}\right) \exp \left(-\alpha \frac{E^{2}}{E_{\mathrm{Pl}}^{2}}\right) \quad g_{2}\left(E / E_{\mathrm{Pl}}\right)=1,
$$

with $\alpha>0$ and $\beta \in \mathbb{R}$, the ZPE of Eq.(2.9) is finite for the pure "Gaussian" choice, $(\beta=0)$ but it does not work correctly because it does not lead to a positive induced cosmological constant. On the other hand the "Non-Gaussian" choice works correctly especially for a de Sitter, Anti de Sitter and Minkowski background. To fix ideas, for a de Sitter background we have $b(r)=\Lambda_{d S} r^{3} / 3$ with $\Lambda_{d S}>0$. Thus Eq.(2.9) leads to the following behavior of $\Lambda /(8 \pi G)$

$$
\frac{\Lambda}{8 \pi G} \simeq\left\{\begin{array}{c}
-\frac{4 \alpha^{5 / 2}+3 \sqrt{\pi} \beta \alpha^{2}}{4 \pi^{2} \alpha^{9 / 2}} E_{P}^{4} x \rightarrow 0 \\
E_{P}^{4} \exp \left(-\alpha x^{2}\right) \quad x \rightarrow \infty
\end{array} .\right.
$$

By imposing that

$$
\beta=-\frac{4}{3} \sqrt{\frac{\alpha}{\pi}}
$$

$\Lambda /(8 \pi G)$ vanishes for small $x$ and therefore the result is regular for every value of $x$, where $x=$ $\sqrt{m_{0}^{2}(r) / E_{P}^{2}}$ and where $m_{0}^{2}(r)$ play the rôle of an effective mass with

$$
m_{0}^{2}(r)=\frac{6}{r^{2}}-\Lambda_{d s}
$$

This "dynamical induced cosmological constant" is finite for every choice of $r$. It is interesting to note that the whole setting successfully applies also to the case of a naked singularity[10] of the form (2.5) with $b(r)=-2 M G, M>0$. One finds that if we define

$$
x=\sqrt{\frac{m_{1}^{2}(r)}{E_{P}^{2}}}=\frac{1}{r E_{P}} \sqrt{6+\frac{15 M G}{r}} \quad \text { and } \quad y=\sqrt{\frac{m_{2}^{2}(r)}{E_{P}^{2}}}=\frac{1}{r E_{P}} \sqrt{6+\frac{9 M G}{r}},
$$


then

$$
\lim _{\substack{x \rightarrow 0 \\ y \rightarrow 0}} \frac{\Lambda}{8 \pi G E_{P}^{4}} \simeq-\frac{4+3 \sqrt{\pi / \alpha} \beta}{4 \pi^{2} \alpha^{2}}=0
$$

if we set

$$
\beta=-\frac{4}{3} \sqrt{\frac{\alpha}{\pi}}
$$

On the other hand, when $r \rightarrow 0$ or $M \rightarrow \infty$, one gets

$$
\frac{\Lambda}{8 \pi G E_{P}^{4}} \simeq \frac{2}{3 \pi^{2} \alpha r^{3} E_{P}^{2}}\left[15 M G \exp \left(-\frac{\alpha 15 M G}{r^{3} E_{P}^{2}}\right)+9 M G \exp \left(-\frac{\alpha 9 M G}{r^{3} E_{P}^{2}}\right)\right] .
$$

The case in which $M \rightarrow \infty$ is unphysical because it represents a singularity which fills the whole universe. On the other hand the case in which $r \rightarrow 0$ represents a naked singularity which is no more singular, at least from the ZPE point of view. Note that the choice (2.12) has been borrowed by Noncommutative Geometry (NCG). In NCG, one introduces a deformation of the classical Liouville counting number of nodes

$$
d n=\frac{d^{3} \vec{x} d^{3} \vec{k}}{(2 \pi)^{3}}
$$

with[12]

$$
d n_{i}=\frac{d^{3} \vec{x} d^{3} \vec{k}}{(2 \pi)^{3}} \exp \left(-\frac{\theta}{4} k_{i}^{2}\right)
$$

where

$$
k_{i}^{2}=E_{i}^{2}-m_{i}^{2}(r) \quad i=1,2 .
$$

$\theta$ is the parameter encoding the noncommutativity of spacetime represented by the commutator $\left[\mathbf{x}^{\mu}, \mathbf{x}^{v}\right]=i \theta^{\mu v} . \theta^{\mu v}$ is an antisymmetric matrix which determines the fundamental discretization of spacetime. However, since Gravity's Rainbow depends on $g_{1}\left(E / E_{\mathrm{Pl}}\right)$ and $g_{2}\left(E / E_{\mathrm{Pl}}\right)$ and not only by one single parameter like $\theta$, it appears to be more flexible in describing ZPE calculations. This flexibility appears more evident when one tries to make a bridge between Gravity's Rainbow and HL theory. Indeed it is possible to create a correspondence between them at least for a FLRW metric and for a spherically symmetric background[13]. This is possible when

$$
\begin{aligned}
& g_{1}\left(E / E_{P}\right) \equiv g_{1}\left(E(a(t)) / E_{P}\right) \\
& g_{2}\left(E / E_{P}\right) \equiv g_{2}\left(E(a(t)) / E_{P}\right)
\end{aligned}
$$

for a FLRW metric and

$$
\begin{aligned}
& g_{1}\left(E / E_{P}\right) \equiv g_{1}\left(E(b(r)) / E_{P}\right) \\
& g_{2}\left(E / E_{P}\right) \equiv g_{2}\left(E(b(r)) / E_{P}\right),
\end{aligned}
$$

for a spherically symmetric metric. This interesting connection gives a hint on why Gravity's Rainbow produces finite results, at least in one loop calculations. 


\section{References}

[1] B. S. DeWitt, Phys. Rev. 160, 1113 (1967).

[2] R. Garattini, Phys. Rev. D 86, 123507 (2012); ArXiv:0912.0136 [gr-qc].

[3] R. Garattini and M. De Laurentis, in preparation.

[4] R. Garattini, TSPU Vestnik 44 N 7, 72 (2004), ArXiv:gr-qc/0409016; R. Garattini, J. Phys. A 39, 6393 (2006), ArXiv:gr-qc/0510061; R. Garattini, J. Phys. Conf. Ser. 33, 215 (2006), ArXiv:gr-qc/0510062. R. Garattini, J. Phys. A 41 (2008) 164057, ArXiv:0712.3246 [gr-qc]; R. Garattini, AIP Conf. Proc. 1241866 (2010), ArXiv: 0911.2393 [gr-qc]. S. Capozziello and R. Garattini, Class.Quant.Grav. 24, 1627 (2007); ArXiv: 0702075 [gr-qc].

[5] P. Hǒrava, JHEP, 0903, 020 (2009). ArXiv: 0812.4287 [hep-th]; P. Hǒrava, Phys. Rev. Lett. 102, 161301 (2009) ArXiv: 0902.3657 [hep-th]. P. Hǒrava, Phys. Rev. D 79, 084008 (2009). ArXiv: 0901.3775 [hep-th].

[6] J. Magueijo and L. Smolin, Class. Quant. Grav. 21, 1725 (2004). ArXiv: 0305055 [gr-qc].

[7] A.DeBenedictis, R. Garattini and F. S.N. Lobo, Phys.Rev. D 78, 104003 (2008), ArXiv:0808.0839 [gr-qc].

[8] R. Garattini and G. Mandanici, Phys. Rev. D 83, 084021 (2011); ArXiv:1102.3803 [gr-qc].

[9] R. Garattini, Phys. Lett. B 685, 329 (2010), ArXiv: 0902.3927 [gr-qc]. R. Garattini and G. Mandanici, Phys. Rev. D 85, 023507 (2012); ArXiv:1109.6563 [gr-qc]. R. Garattini, JCAP 1306, 017 (2013), ArXiv: 1210.7760 [gr-qc]. R. Garattini and F. S. N. Lobo, Phys. Rev. D 85, 024043 (2012), ArXiv:1111.5729 [gr-qc]. R. Garattini, Int. J. Mod. Phys. Conf. Ser. 14, 326 (2012), ArXiv:1112.1630 [gr-qc]. R. Garattini and F.S.N. Lobo, Eur. Phys. J. C 74 (2014), ArXiv:1303.5566 [gr-qc]. R. Garattini and B. Majumder, Nucl. Phys. B 883 (2014), ArXiv:1305.3390 [gr-qc]. R. Garattini and M. Sakellariadou, Phys. Rev. D 90043521 (2014), ArXiv:1212.4987 [gr-qc].

[10] R. Garattini and B. Majumder, Nucl. Phys. B 884, (2014), ArXiv:1311.1747 [gr-qc].

[11] Y. Ling, JCAP 17, 708 (2007), gr-qc/0609129; Y. Ling, X. Li and H. Zhang, Mod.Phys.Lett. A 22, 2749 (2007), gr-qc/0512084; Y. Ling, B. Hu and X. Li, Phys. Rev. D 73, 087702 (2006), gr-qc/0512083.

[12] R. Garattini and P. Nicolini, Phys. Rev. D 83, 064021 (2011); ArXiv:1006.5418 [gr-qc].

[13] R. Garattini and E. N. Saridakis, Gravity's Rainbow: a bridge towards Horava-Lifshitz gravity; ArXiv:1411.7257. 